\title{
Efecto de dosis altas de budesonida y función suprarrenal de niños asmáticos severos
}

\author{
Aída Milinarsky $\mathrm{T}^{1}$, Sylvia Fischer $\mathbf{G}^{2}$, Vinka Giadrosich $\mathrm{R}^{1}$, Viviana \\ Lezana $\mathbf{S}^{\mathbf{1}}$, María Teresa Torres $\mathbf{C}^{\mathbf{1}}$. \\ High doses of inhaled budesonide and adrenal \\ function in children with severe asthma
}

\begin{abstract}
Background: The use of inhaled steroids is common in the treatment of bronchial asthma in children. Aim: To assess adrenocortical function in children with severe asthma receiving inhaled budesonide for six or more months. Material and methods: Children with severe asthma that required $800 \mu \mathrm{g} /$ day or more of inhaled budesonide and that did not required systemic steroids for more than six days in the last four months to control their disease, were studied. Serum cortisol was measured one hour after administration of $0.25 \mathrm{mg}$ of ACTH (Cosyntropin ${ }^{\circledR}$ ) intravenously. The test was considered normal if post stimulation serum cortisol was over $18 \mu \mathrm{g} / \mathrm{ml}$. Results: Twenty children (aged 5 to 14 years, 15 males), were studied. The stimulation test was normal in 17 children. Conclusions: Alterations in adrenal function are present in a small proportion of asthmatic children who require $800 \mu \mathrm{g} /$ day of inhaled budesonide (Rev Méd Chile 2006; 134: 60-4).
\end{abstract}

(Key words: Adrenal cortex function tests; Asthma; Budesonide; Hydrocortisone)

Recibido el 9 de septiembre, 2004. Aceptado el 15 de julio, 2005.

${ }_{1}^{1}$ Departamento de Pediatría, Escuela de Medicina, Universidad de Valparaíso.

${ }^{2}$ Departamento de Medicina, Escuela de Medicina, Universidad de Valparaíso.

$\mathrm{E}$ uso de corticoides inhalados (CI) es considerado la terapia antiinflamatoria de elección en asma bronquial ${ }^{1,2}$. La mayoría de los niños responden a dosis habituales de corticoides inhalados, pero hay un grupo de pacientes que requieren dosis más altas para el adecuado control de su enfermedad.

Entre los efectos sistémicos colaterales que pueden producir los corticoides, se describe la supresión suprarrenal ${ }^{3}$. El nivel suprafisiológico que alcance el cortisol plasmático es capaz de inhibir el eje hipotálamo-hipófisis-suprarrenal ${ }^{4}$. Como consecuencia de ello, el hipocortisolismo puede manifestarse clínicamente con anorexia, náusea, dolor abdominal, hipoglicemia, crisis convulsivas, shock y muerte ${ }^{5}$.

Correspondencia a: Dra. Aída Milinarsky. Departamento de Pediatría, Escuela de Medicina, Universidad de Valparaíso. Hontaneda 2653, Valparaíso, Chile. Fono: 507358. Fax: 507360. E mail: pediatria@uv.cl
La vía inhalatoria ha sido utilizada con el fin de evitar los efectos sistémicos de los corticoides, ya que su absorción senía mínima, sin embargo, la literatura señala resultados discordantes. Volovitz ${ }^{5}$, en 1993, evaluó 15 niños con asma severo que fueron tratados con budesonida por más de tres años y no encontró compromiso de función supramenal, medida por test de ACTH, cortisol matinal y cortisol libre urinario. Kannisto ${ }^{6}$, en un grupo de 60 pacientes asmáticos tratados con corticoides inhalados: 30 con fluticasona en dosis de $500 \mu \mathrm{g} /$ día por 2 meses y luego 200 y 30 pacientes con budesonida en dosis de 800 y luego 400 y $200 \mu \mathrm{g} /$ día, encontró compromiso moderado de la función suprarrenal en $23 \%$ de los pacientes. Todd ${ }^{7}$ realizó un rastreo mediante un cuestionario enviado a pediatras y endocrinólogos, identificando a 28 niños y 5 adultos de un total de 2.912 pacientes que presentaron síntomas de hipoglicemia, compromisos variables de conciencia o convulsiones, y 1 paciente llegó al 
coma y muerte. $\mathrm{Sim}^{8}$, en un estudio de 50 niños tratados con dosis altas de fluticasona $(1.000 \mathrm{mg} /$ día por más de 6 meses) encontró en $12 \%$ de los pacientes, evidencias bioquímicas de insuficiencia suprarrenal. Bacharier ${ }^{9}$ no encontró compromiso suprarrenal en 63 pacientes asmáticos tratados con budesonida en dosis habitual. Mahachoklertwattana ${ }^{10}$ evaluó a 18 niños asmáticos de 7 a 17 años tratados con fluticasona en dosis de 250-750 mg/día y encontró que $50 \%$ de ellos presentaron supresión suprarrenal, evaluada mediante prueba de respuesta suprarrenal a hipoglicemia inducida por insulina.

Es importante reconocer a los pacientes en riesgo de presentar insuficiencia suprarrenal aguda ante alguna situación crítica. El método recomendado para evaluar reserva suprarrenal es la determinación de cortisol bajo exigencia, como es el estímulo de corticotropina (ACTH) ${ }^{4}$.

El propósito de este trabajo fue evaluar el impacto de $800 \mu \mathrm{g}$ de budesonida, por 6 o más meses, en la función suprarrenal de niños asmáticos severos.

\section{PACIENTES Y MÉTODO}

Pacientes. En un estudio descriptivo prospectivo se consideraron niños asmáticos severos (de acuerdo al criterio de la American Thoraxic Society) ${ }^{1}$, cuyos padres y ellos aceptaron participar en el estudio. Estos pacientes fueron reclutados del Policlínico de Enfermedades Respiratorias Infantiles del Hospital Carlos Van Buren de Valparaíso y del Instituto Médico Infantil de Viña del Mar.

Se seleccionó a 20 pacientes consecutivos que cumplieran con los siguientes requisitos: diagnóstico de asma bronquial severa, entre 1 y 3 años de evolución. Requerir dosis de $800 \mu \mathrm{g}$ al día de budesonida por 6 o más meses para estabilizar su asma; no haber requerido tratamiento con corticoides sistémicos por más de 6 días consecutivos en los últimos 4 meses (dosis diaria $1 \mathrm{mg} / \mathrm{kg}$ de peso, máximo $30 \mathrm{mg} /$ día); con un total de no más de 20 días en los últimos 6 meses. Saber utilizar en forma adecuada el turbohaler, incluyendo un buen lavado de la boca después de su uso. No tener otra enfermedad sistémica crónica. Se descartaron los pacientes portadores de otra patología crónica no respiratoria.

Método. El cortisol plasmático se midió con kit comercial RIA Coat-a-count DPC. Intervalo de calibración: 1-50 g/dl. Sensibilidad analítica: 0,2 $\mu \mathrm{g} / \mathrm{dl}$ (Tabla 1 y 2 ).

El test de estimulación suprarrenal se realizó en ayunas entre 8 y 9 AM y consistió en determinar los valores de cortisol, 1 hora después de la administración intravenosa de 0,25 mg de ACTH

Tabla 1. Precisión intra-ensayo ( $\mu \mathrm{g} / \mathrm{dl})$

\begin{tabular}{|cccc|}
\hline & Promedio cortisol $(\mu \mathrm{g} / \mathrm{dl})$ & Desviación estándar & Coeficiente variación \% \\
\hline 1 & 3,1 & 0,15 & 4,8 \\
2 & 4,5 & 0,19 & 4,2 \\
3 & 9,9 & 0,47 & 4,7 \\
4 & 12,0 & 0,61 & 5,1 \\
5 & 20,0 & 0,60 & 3,0 \\
6 & 25,0 & 1,00 & 4,0 \\
7 & 34,0 & 1,50 & 4,4 \\
\hline
\end{tabular}

Tabla 2. Precisión inter-ensayo ( $\mu \mathrm{g} / \mathrm{dl})$

\begin{tabular}{|cccc|}
\hline & Promedio cortisol $(\mu \mathrm{g} / \mathrm{dl})$ & Desviación estándar & Coeficiente variación $\%$ \\
\hline 1 & 3,3 & 0,17 & 5,2 \\
2 & 21,0 & 0,83 & 4,0 \\
3 & 36,0 & 2,30 & 6,4 \\
\hline
\end{tabular}


(Cosyntropin, nombre comencial Synacthen ${ }^{\circledR}$ de Laboratorio Novartis). El cosyntropin utilizado es la molécula idéntica en los primeros 24 aminoácidos, de los 39 del ACTH natural. Es de acción rápida y de uso endovenoso. La dosis de $250 \mathrm{mcg}(0,25 \mathrm{mg})$ de cosyntropin equivale a 25 unidades USP de ACTH. El cortisol plasmático basal se comparó con valores normales de referencia de Lashansky ${ }^{12}$ considerándose normal sobre $5 \mu \mathrm{g} / \mathrm{dl}$. El test de estimulación suprarrenal fue considerado normal si los niveles de cortisol después de administrar ACTH superaban los $18 \mu \mathrm{g} / \mathrm{dl}^{12}$.

Este trabajo fue aprobado por el Comité de Ética de la Escuela de Medicina de la Universidad de Valparaíso.

\section{RESULTADOS}

Los niños tenían entre 5 y 14 años: 15 varones y 5 niñas.

Antes de incorporarse al presente estudio, estos niños habían usado broncodilatadores, corticoides inhalados en forma irregular y corticoides sistémicos ocasionalmente dentro de los márgenes planteados en el protocolo de selección.

El promedio de Z score de talla fue 0,235 (DS 1.01). El promedio de $Z$ score de IMC fue 1,085 (DS 1,12).

Al examen físico no hubo pacientes con signos clínicos de Cushing. No se pesquisaron fracturas patológicas en el período de observación.

Tabla 3. Test de ACT H sintético en 20 niños asmáticos severos tratados con altas dosis de budesonida

\begin{tabular}{|c|c|c|c|c|c|c|}
\hline Paciente & $\begin{array}{l}\text { Edad } \\
\text { (años) }\end{array}$ & $\mathrm{Z}$ de IMC & $\mathrm{Z}$ de talla & $\begin{array}{c}\text { Sexo } \\
(\mathrm{M} / \mathrm{F})\end{array}$ & $\begin{array}{l}\text { Cortisol } \\
\text { plasmático } \\
\text { basal } \\
(\mu \mathrm{g} / \mathrm{dl})\end{array}$ & $\begin{array}{c}\text { Cortisol } \\
\text { plasmático } \\
\text { post ACTH } \\
(\mu \mathrm{g} / \mathrm{dl})\end{array}$ \\
\hline 1 & 7 & 0,7 & 0,19 & M & 10,78 & 39,78 \\
\hline 2 & 9 & $-0,4$ & 0,80 & M & 13,34 & 51,92 \\
\hline 3 & 11 & 3,0 & $-0,36$ & $\mathrm{~F}$ & 9,64 & 31,78 \\
\hline 4 & 11 & 1,9 & 1,18 & $\mathrm{M}$ & 20,54 & 37,11 \\
\hline 5 & 9 & 1,1 & 0,00 & $\mathrm{~F}$ & 12,55 & 33,01 \\
\hline 6 & 5 & 0,9 & $-0,36$ & M & 4,23 & 27,41 \\
\hline 7 & 5 & 0,4 & $-1,34$ & M & 5,53 & 27,86 \\
\hline 8 & 6 & 1,6 & 1,18 & M & 19,35 & 34,59 \\
\hline 9 & 6 & $-1,2$ & $-0,74$ & M & 18,74 & 36,41 \\
\hline 10 & 9 & 1,3 & $-1,90$ & M & 1,39 & $8,29 *$ \\
\hline 11 & 6 & 1,8 & 1,32 & $\mathrm{~F}$ & 12,17 & 37,95 \\
\hline 12 & 9 & 1,9 & 1,71 & M & 17,06 & 35,34 \\
\hline 13 & 7 & 0,5 & 0,20 & M & 17,63 & 32,56 \\
\hline 14 & 10 & 1,7 & 1,43 & $\mathrm{M}$ & 3,86 & 27,62 \\
\hline 15 & 14 & 2,4 & 0,20 & $\mathrm{M}$ & 12,44 & 29,92 \\
\hline 16 & 6 & 1,0 & 0,00 & $\mathrm{M}$ & 18,39 & 36,99 \\
\hline 17 & 11 & $-1,1$ & 1,80 & $\mathrm{M}$ & 17,40 & 35,51 \\
\hline 18 & 10 & 0,7 & $-0,40$ & $\mathrm{~F}$ & 17,18 & 35,32 \\
\hline 19 & 12 & 0,8 & 0,39 & $\mathrm{M}$ & 7,16 & $14,84^{*}$ \\
\hline 20 & 8 & 2,7 & $-0,60$ & $\mathrm{~F}$ & 6,69 & $11,49 *$ \\
\hline Promedios & 8,55 & 1,085 & 0,235 & & 12,30 & 31,29 \\
\hline Desviación estándar & & 1,12 & 1,01 & & 5,92 & 10,09 \\
\hline
\end{tabular}

(*) Respuesta insuficiente. 
Dieciséis niños tuvieron cortisol plasmático normal en ayunas y adecuada respuesta a la estimulación con ACTH.

El promedio de cortisol plasmático basal fue 12,30 $\mu \mathrm{g} / \mathrm{dl}$ y su desviación estándar (DS) 5,9 $\mu \mathrm{g} /$ dl. El valor promedio de cortisol plasmático post ACTH fue $31,29 \mu \mathrm{g} / \mathrm{dl}$ y su DS 10,09. Tres pacientes no superaron los $18 \mu \mathrm{g} / \mathrm{dl}$ de cortisol plasmático una hora después de recibir ACTH intravenoso. Uno de ellos tenía además cortisol plasmático basal muy bajo. Los otros 2 pacientes tenían cortisol basal en rangos normales.

Hubo otros 2 pacientes con valor de cortisol basal bajo y que respondieron bien a la estimulación con ACTH (Tabla 3).

\section{DisCUSIÓN}

Dado que el asma bronquial se ha considerado como una enfermedad inflamatoria crónica de la vía aérea, los CI son la terapia de elección ${ }^{1-3}$, sin embargo, hay preocupación acerca de la seguridad de su uso a corto y largo plazo, lo que puede contribuir a su subutilización ${ }^{3}$. En este estudio, demostramos que la terapia con budesonida inhalada en dosis de $800 \mu \mathrm{g} /$ día por seis 0 más meses, se asoció con alteración de la respuesta del eje hipotálamo-hipófisis-suprarrenal a la estimulación con ACTH en 3 de 20 niños asmáticos severos.

El estudio se realizó con pacientes asmáticos severos. La condición de asma severo exige tratamiento con corticoides, por lo que no se pudo disponer de un grupo de pacientes asmáticos severos sin tratamiento como control.

La evaluación del efecto de supresión del eje hipotálamo-hipófisis-suprarrenal causado por dosis suprafisiológicas de corticoides puede realizarse a través de diferentes pruebas de laboratorio. Se ha propuesto el test de estimulación con ACTH, la determinación del área bajo la curva de las concentraciones seriadas de cortisol sérico y excreción del cortisol libre urinario de $24 \mathrm{~h}^{13,14}$ entre otros.
La determinación del área bajo la curva de las concentraciones seriadas de cortisol sérico y el cortisol libre urinario de 24 horas aportan información del estado cotidiano de la función suprarrenal. Elegimos la prueba de estimulación con ACTH intravenoso con el fin de detectar la capacidad de respuesta de la glándula suprarrenal frente a una estimulación importante, como podría ser un evento crítico. Otro método que cumple la misma función es el test de tolerancia a la insulina, que no se eligió por los riesgos que implica $^{10}$.

La prueba de estimulación con ACTH se realizó con la dosis estándar de $250 \mu \mathrm{g} / 1,73 \mathrm{~m}^{2}$, porque una dosis más baja $\left(0,5-1 \mu \mathrm{g} / 1,73 \mathrm{~m}^{2}\right)$ no está aún universalmente aceptada ${ }^{13-15}$. Es posible que hayamos omitido algunos casos de insuficiencia suprarrenal leve, que no serían detectadas por las dosis altas de ACTH empleadas; sin embargo, es necesario considerar que alteraciones bioquímicas de pequeña magnitud, no siempre pueden tener importancia clínica.

Considerando que hubo compromiso de la función suprarrenal evidenciada por prueba bioquímica en 3 de los 20 niños estudiados, sólo podemos decir que 1 de ellos tenía talla bajo lo normal para su edad, aclarando además que los padres son de talla baja. Este resultado de prueba de laboratorio nos plantea la inquietud acerca de la respuesta clínica que puedan tener estos niños frente a un eventual estrés. Sugerimos que frente a un paciente que está requiriendo dosis altas de CI para el control de su asma haya un control sobre efectos colaterales de corticoides. Habría que aumentar el tamaño de la muestra, para tratar de llegar a resultados más concluyentes, para encontrar algún signo o antecedente clínico que haga sospechoso de presentar alguno de estos efectos indeseados de los corticoides, ya que efectuar trabajo con grupo placebo, no sería posible por razones éticas.

En conclusión, el tratamiento con $800 \mu \mathrm{g}$ de budesonida al día, por 6 o más meses, en niños asmáticos severos puede deprimir la función suprarrenal en algunos casos. 


\section{REFERENCIAS}

1. National Institutes of Health, National Heart, Lung, and Blood Institute. Executive Summary of the NAEPP Expert Panel Report-Guidelines for the Diagnosis and Management of Asthma-Update on Select topics. 2002. Bethesda, MD:NIH; 2002. (Publication № 02-5075)

2. BARNES P. Review articles: Drug therapy: Inhaled glucocorticoids for asthma. N Engl J Med 1995; 332: 868-75.

3. EvERITT BJ. Inhaled corticosteroids in asthma: Should I or shouldn't I? Curr Opin Invest Drugs 1993; 2: 843-50.

4. Aron D, Finding J, Tyrrell JB. Hipotálamo e hipófisis. Endocrinología básica y clínica. Greenspan y Gardner. 5a edición 2003. Editorial El Manual Moderno. México DF. página 123.

5. Volovitz B, Amr J, Malik H, Kauschansky A, VARSANO I. Growth and pituitary-adrenal function in children with severe asthma treated with inhaled budesonide. N Engl J Med 1993; 329: 1703-8.

6. Kannisto S, Korppi M, Remes K, Voutilainen R. Adrenal suppression, evaluated by a low dose adrenocorticotropin test and growth in asthmatic children treated with inhaled esteroids. J Clin Endocrinol Metab 2000; 85: 652-7.

7. Todd GRG, Acerini CL, Ross-Russel R, Zahra S, WarNer JT, McCance D. Survey of adrenal crisis associated with inhaled corticoisteroids in the United Kingdom. Arch Dis Child 2002; 87: 457-61.

8. Sim D. Adrenal suppression from high-dose inhaled fluticasone propionate in children with asthma. Eur Respir J 2003; 21(4): 633-6.

9. Bacharier L, Raissy H, Wilson L, McWimams B, Strunk R, KeLLY W. Long-Term Effect of Budesoni- de on Hypothalamic-Pituitary-Adrenal -Axis in Children with Mild to Moderate Asthma. Pediatrics 2004; 113: 1693-9.

10. Mahachokiertwattana $P$, Sudkronrayudh K, Direkwattanachai C, Choubtun L, Okascharoen C. Dedreased cortisol response to insulin induced hypoglycaemia in asthmatics treated with inhaled fluticasone propionate. Arch Dis Child 2004; 89: 1055-8.

11. Chrousos GP, HaRRIS AG. Hypothalamic-pituitaryadrenal axis suppression and inhaled corticosteroid therapy. 1. General principles. Neuroimmunomodulation 1998; 5: 277-87.

12. Lashansky G, Saenger P, Fishman K et al. Normative data for adrenal steroidogenesis in a healthy pediatric population: age-and sex-related changes after adrenocorticotropin stimulation. J Clin Endocrinol Metab 1991; 73: 674-86.

13. Crowiey S, Hindmarsh PC, Honour JW, Brook CG. Reproducibility of the cortisol response to stimulation with a low dose of ACTH (1-24): the effect of basal cortisol levels and comparison of lowdose with high-dose secretory dynamics. J Endocrinol 1993; 136: 167-72.

14. Broide J, Soferman R, Kivity S, Golander A, Dickstein G, Sirirer Z, Weisman Y. Low-dose adrenocorticotropin test reveals impaired adrenal function in patients taking inhaled corticosteroids. J Clin Endocrinol Metab 1995; 80: 1243-6.

15. Karlsson R, Kawo J, Toppari J, Kero P. Timing of peak serum cortisol values in preterm infants in low-dose and the standard ACTH tests. Pediatr Res 1999; 45: 367-9.

Agradecimientos

Los autores agradecen a don Carlos Cacciuttolo por su gentil colaboración con las pruebas de laboratorio y a Gabriela González como secretaria. 